\title{
Riesgo de Sodicidad en los Suelos de Cantón Milagro, Guayas- Ecuador en Época de Estiaje
}

\author{
Pérez Molina, Alexandra ${ }^{1}$ \\ ${ }^{1}$ Universidad de las Fuerzas Armadas - ESPE, Departamento de Ciencias Exactas, Sangolquí, Ecuador
}

\begin{abstract}
Resumen: Dada la importancia de la producción agrícola del suelo del cantón Milagro, en este artículo se plantea como objetivo estudiar el efecto de las bajas precipitaciones y el uso de riego complementario, en la concentración de sales en los suelos del cantón. El problema de las inundaciones y la sequía ponen en riesgo las actividades agrícolas de la zona que ocupan un $95,5 \%$ del suelo del cantón, ya que pueden provocar la pérdida de suelos fértiles . La determinación del RAS relación de adsorción de sodio en todas sus modificaciones, y el Î́ndice de Saturación en los suelos, calculados a partir de las mediciones de la concentración de cationes, aniones, conductividad eléctrica (CE) y $\mathrm{pH}$ en muestras de aguas tomadas en 32 estaciones a lo largo de la microcuenca del río Milagro a juicio del experto y para los fines específicos de la investigación, en junio de 2013 con un promedio de precipitaciones de 74,5 mm, permitirá proponer alternativas para evitar la sodicidad de los suelos del cantón. En estudios realizados sobre la relación agua-suelo se ha demostrado que es posible predecir el riesgo de sodicidad de los suelos por el uso de aguas de alta salinidad, como es el caso de algunas muestras de aguas en el cantón Milagro. Los valores más críticos se obtuvieron con el RAS $\mathrm{aj}_{\mathrm{aj}}$, el río Belin tiene una clasificación S2 según Richard et. al. (1959), aguas provenientes de bananeras, lo que significa que la precipitación de la calcita es un factor influyente, son zonas de conos de esparcimiento. Existen zonas clasificadas como S3 y S4 en la zona de Banco de Arena, situación que indica una elevada cantidad de sodio en el agua de riego lo que puede causar que los suelos alcancen un nivel peligroso de sodio y para una agricultura sostenible se requiere de lavados y un muy buen drenaje.
\end{abstract}

Palabras clave: riego, precipitaciones, índice de saturación, sodicidad y relación de adsorción de sodio RAS.

\section{Risk of Sodicity in the Soils of Canton Milagro, Guayas-Ecuador at Time of Dryness}

\begin{abstract}
Given the importance of the agricultural production of the soil of the canton Milagro, in this article the objective is to study the effect of low rainfall and the use of complementary irrigation in the concentration of salts in the soils of the canton. The problem of floods and drought endangers agricultural activities in the area that occupy $95.5 \%$ of the land of the canton, as they can cause the loss of fertile soils. The determination of the RAS ratio of sodium adsorption in all its modifications, and the Saturation Index in the soils, calculated from the measurements of the concentration of cations, anions, electrical conductivity (CE) and $\mathrm{pH}$ in water samples taken in 32 stations along the Milagro river microbasin, according to the expert and for the specific purposes of the investigation, in June 2013 with an average rainfall of $74.5 \mathrm{~mm}$, will allow to propose alternatives to avoid the sodicity of the soil of the canton. In studies carried out on the water-soil relationship it has been shown that it is possible to predict the risk of sodicity of soils by the use of high salinity water, as is the case of some water samples in the Milagro canton. The most critical values were obtained with the RASaj, the Belin River has an S2 classification according to Richard et. to the. (1959), waters from banana plantations, which means that the precipitation of calcite is an influencing factor, they are zones of recreational cones. There are zones classified as S3 and S4 in the area of Banco de Arena, a situation that indicates a high amount of sodium in the irrigation water which can cause the soils to reach a dangerous level of sodium and for a sustainable agriculture requires washing and a very good drainage.
\end{abstract}

Keywords: irrigation, precipitation, saturation index, sodicity and sodium adsorption ratio RAS.

\section{INTRODUCCIÓN}

La reutilización del agua como aguas para regadío, especialmente en época de estiaje, es una de las mayores preocupaciones en la actualidad, dado que el alto contenido de sales de éstas puede producir la salinidad de los suelos que sustentan las plantas afectando la productividad de los cultivos. Esta contaminación va más allá de los suelos pues está en juego también la contaminación de las aguas subterráneas. 
El comportamiento de las aguas para riego se ve modificado por algunos factores entre los que podemos mencionar las condiciones climáticas que afectan el sector de análisis. De acuerdo a CLIRSEN (2009) el cantón Milagro se encuentra en una zona de déficit hídrico para la producción agrícola, en consecuencia el uso de aguas de riego es necesario, más aún en épocas de estiaje en donde la precipitación anual es insuficiente para satisfacer las necesidades de evapotranspiración de los cultivos, lo que no permite la solubilidad de las sales del suelo.

El cantón Milagro ubicado en la provincia del Guayas Ecuador, es una de las ciudades de mayor progreso en la provincia, debido a su intensidad comercial y el desarrollo de su industria agro-productiva, se encuentra el $61,91 \%$ que corresponde a 24949,00 hectáreas de su superficie con riego; esta superficie se localiza principalmente en el norte y centro del territorio cantonal y en partes del sector sur del mismo; la superficie con riego se conforma con las plantaciones de arroz, caña de azúcar industrial y banano, que debido a la importancia económica de los mismos se los provee con algún sistema de riego. El banano tiene riego por aspersión, la caña de azúcar tiene riego por gravedad y el arroz se riega por inundación. Existen 13 526,56 hectáreas que corresponden al 33,57\% del territorio del Cantón Milagro, que no tienen ningún tipo de riego; esta superficie se encuentra localizada de manera dispersa en el centro y sur del territorio y está ocupada por cultivos de cacao con sombra y sin sombra, pasto cultivado, maíz, soya y plátano principalmente (PDOTM, 2014). El área de cultivo alcanza el 95,5\%, según CLIRSEN (2009), de producción agrícola permanente del cantón, por ello se hace prioritario conocer cómo están afectados los suelos por el tipo de agua utilizada para riego, en especial en la época de estiaje, comprendida entre los meses de junio y diciembre según los reportes de la Estación Meteorológica de Milagro M072 (INAMHI, 2013).

Un estudio realizado en el noreste de China correlaciona el efecto de la salinidad de las aguas de riego con la composición de los suelos, dada la estrecha relación suelo-agua. El estudio desarrolló un modelo matemático que permite predecir la calidad del suelo a partir de datos de fácil medición en las aguas (Chen, 2010). Sin embargo, el deterioro del suelo no solo depende de la calidad del agua usada para riego, sino también de las propias características del suelo. La salinidad del agua de riego determina la salinidad de los suelos que afectan a los cultivos. Los Sólidos Totales Disueltos es un parámetro de suelos y agua de riego para determinar su calidad y su efecto sobre los cultivos (Torres, 2008).

\section{MATERIALES Y MÉTODOS}

El cantón Milagro se encuentra ubicado aproximadamente a 40 kilómetros de la ciudad de Guayaquil en la provincia del Guayas, en las coordenadas geográficas $1^{\circ} 59^{\prime}$ a $2^{\circ} 14^{\prime}$ de latitud Sur y de $79^{\circ} 41^{\prime}$ a $79^{\circ} 27^{\prime}$ de longitud Oeste, Figura 1. Tiene una extensión territorial de 405,63 km², y sus límites son: al norte con los cantones Yaguachi, Baquerizo Moreno y Simón Bolívar, al sur con los cantones Yaguachi y Marcelino Maridueña, al este con los cantones Naranjito y Marcelino Maridueña; y al oeste con el cantón Yaguachi.
La microcuenca del río Milagro se encuentra inmersa en la cuenca del río Guayas, ocupando el 9,51\% de la subcuenca del río Jujan, 7,04\% de la subcuenca del río Yaguachi y 0,20\% de Drenajes menores (CLIRSEN, 2009), es la que abastece de agua para riego al cantón a través de un sistema de riego público que consta en el plan de desarrollo de la provincia del Guayas 2012 - 202.

Se encuentra en una zona de clima tropical mega térmico húmedo, con una temperatura promedio de $25^{\circ} \mathrm{C}$ y precipitaciones anuales de 1100 a $1800 \mathrm{~mm}$. Milagro se asienta sobre la zona oriental de la unidad morfológica denominada Depresión de Guayas que se ha rellenado paulatinamente con gran cantidad de sedimentos de origen reciente, Holoceno, aportados por algunos volcanes y por la erosión de la cordillera y que paulatinamente fueron arrastrados desde las partes altas por las corrientes de diversos afluentes que finalmente formaron entre otros ríos, el río Milagro. El depósito de estos materiales en la parte baja, formó la gran llanura de inundación donde hoy se asientan varias ciudades como Milagro (PDOTM, 2014).

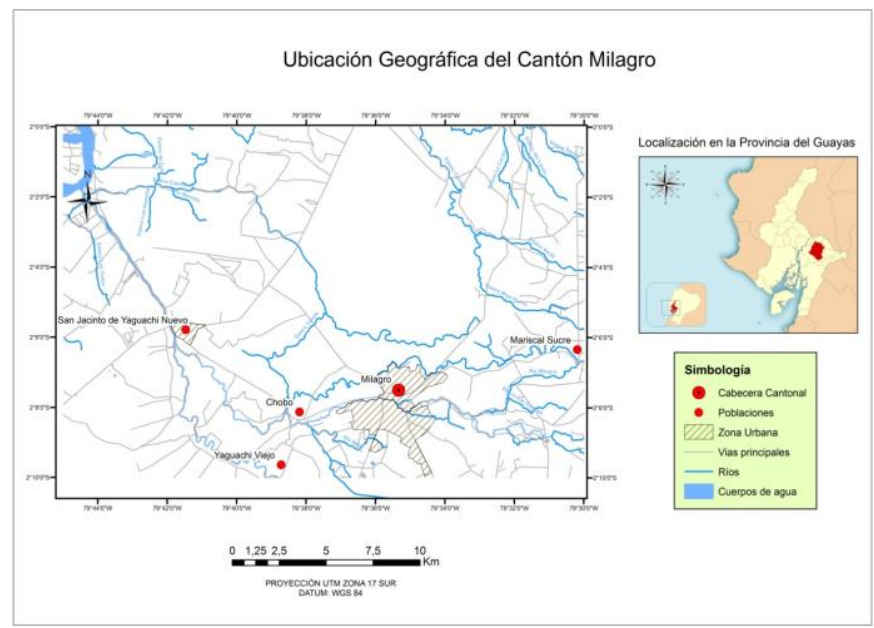

Figura 1. Ubicación geográfica del cantón Milagro. Shapes (IGM, 2009)

La puntos de muestreo se establecieron alrededor de los nacimientos de los ríos, al este del cantón Milagro, en donde se encuentran los sistemas hidrográficos: Belín, Milagro, Los Monos, Tímalo y Carrizal y terminó en los campos en donde se aprovecha esta agua para irrigación. Fueron 32 puntos de muestreo, considerando pozos y manantiales y antes que el río pase por las zonas urbanas y después de las mismas, para conocer los cambios en la salinidad.

Las coordenadas de las estaciones de muestreo fueron determinadas con un GPS (Geografical Position System) y localizadas en las cartas topográficas (IGM, 2009) (1:1 000 000) digitales del río Milagro (Datum WGS-84) de las cuales se tomaron las coordenadas y la altitud aproximada de las márgenes del río. La Figura 2 muestra la ubicación geográfica de los puntos de muestreo. 


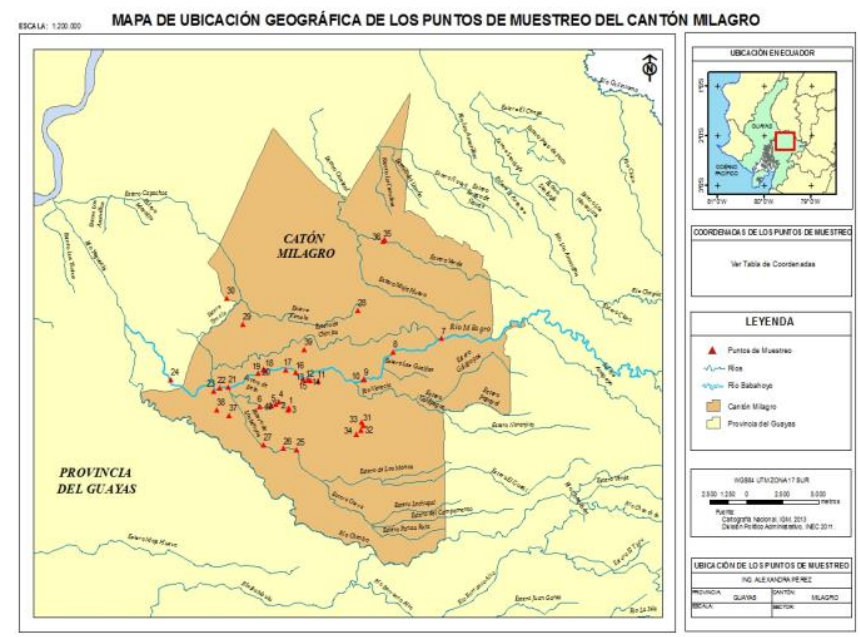

Figura 2. Localización geográfica de los puntos de muestreo del río Milagro

Para asegurar la precisión y fiabilidad de los datos, su interpretación y las acciones a tomar sobre los resultados, se procedió a tomar 1 litro de la muestra en envases de plásticos por su resistencia y poca reactividad con los iones del agua (GYPAN, 2012), debidamente identificados con fecha, lugar y hora de recolección, en dos épocas del año para tener una información representativa en relación a las variaciones de la calidad del agua con las estaciones del año (Lenntech, n.d.).

Se determinó $\mathrm{pH}$, conductividad eléctrica, concentración de cationes y aniones y se calcularon algunos índices como el índice de salinidad IS y relación de adsorción de sodio (RAS), la descripción de las propiedades del agua de riego se detallan en la Tabla 1. Los métodos de análisis para los diferentes parámetros se detallan en la Tabla 2. Todas las figuras de este trabajo fueron realizadas por el autor utilizando la herramienta de interpolación de la extensión espacial Analyst del software ArcGis 9.3, con dos métodos diferentes; uno determinista, exacto y local IWD (media ponderada por el inverso de la distancia) y otro geoestadístico y analítico, kriging ordinario, que tiene en cuenta la autocorrelación espacial de la variable a interpolar.

Tabla 1. Descripción de las propiedades físicas del agua de riego en época de estiaje

\begin{tabular}{|c|c|c|c|c|c|c|c|c|c|c|c|c|c|c|c|c|c|c|c|}
\hline \multirow[b]{2}{*}{ No. } & \multirow[b]{2}{*}{$\mathbf{p H}$} & \multirow{2}{*}{$\begin{array}{c}\text { CE } \\
\mu \mathrm{S} \mathrm{cm}^{-1}\end{array}$} & \multirow{2}{*}{$\begin{array}{c}\mathbf{C E} \\
\mathrm{Ds} \mathrm{m}^{-1}\end{array}$} & \multicolumn{10}{|c|}{ mmol $_{\mathbf{c}} \mathbf{L}^{-1}$} & \multirow{2}{*}{$\begin{array}{c}\mathrm{mg} \mathrm{L}^{-1} \\
\text { STD }\end{array}$} & \multirow{2}{*}{$\begin{array}{c}\% \\
\text { error }\end{array}$} & \multirow[b]{2}{*}{$\mathbf{R A S} \mathbf{S}_{\text {orig }}$} & \multirow[b]{2}{*}{ IS } & \multirow[b]{2}{*}{$\mathbf{R A S}_{a j}$} & \multirow[b]{2}{*}{ RAS } \\
\hline & & & & $\mathrm{Ca}^{2+}$ & $\mathrm{Mg}^{2+}$ & $\mathrm{Na}^{+}$ & $\mathrm{K}^{+}$ & Suma & $\mathrm{CO}_{2}$ & $\mathrm{HCO}_{3}^{-}$ & $\mathrm{Cl}^{-}$ & $\mathrm{SO}_{4}^{2-}$ & Suma & & & & & & \\
\hline 1 & 7,6 & 699,0 & 0,699 & 1,44 & 1,68 & 2,51 & 1,27 & 6,90 & 0,00 & 5,43 & 0,83 & 0,63 & 6,90 & 416 & 0,02 & 2,01 & 6,20 & 14,47 & 0,92 \\
\hline 2 & 7,5 & 334,5 & 0,335 & 0,46 & 0,53 & 1,84 & 0,48 & 3,31 & 0,00 & 0,69 & 2,51 & 0,10 & 3,30 & 250 & 0,18 & 2,62 & 8,40 & 24,58 & 1,76 \\
\hline 4 & 7,5 & 649,0 & 0,649 & 1,33 & 1,52 & 2,40 & 1,16 & 6,41 & 0,00 & 1,93 & 2,55 & 1,93 & 6,41 & 408 & 0,04 & 2,01 & 6,20 & 14,48 & 1,78 \\
\hline 5 & 7,4 & 674,0 & 0,674 & 1,38 & 1,57 & 2,52 & 1,23 & 6,70 & 0,00 & 2,47 & 2,57 & 1,67 & 6,70 & 398 & 0,02 & 2,07 & 6,20 & 14,94 & 1,89 \\
\hline 6 & 7,4 & 781,5 & 0,782 & 1,65 & 1,86 & 2,82 & 1,47 & 7,80 & 0,00 & 2,50 & 2,80 & 2,50 & 7,80 & 464 & 0,00 & 2,13 & 3,59 & 9,76 & 2,07 \\
\hline 7 & 7,6 & 290,0 & 0,290 & 0,65 & 0,66 & 1,08 & 0,52 & 2,91 & 0,00 & 0,14 & 2,62 & 0,14 & 2,90 & 226 & 0,20 & 1,33 & 2,10 & 4,14 & 0,88 \\
\hline 8 & 6,7 & 573,0 & 0,573 & 1,25 & 1,55 & 1,78 & 1,11 & 5,69 & 0,00 & 2,93 & 1,43 & 1,33 & 5,70 & 238 & 0,06 & 1,50 & 3,00 & 6,02 & 1,24 \\
\hline 9 & 7,5 & 290,0 & 0,290 & 0,68 & 0,67 & 1,06 & 0,50 & 2,91 & 0,00 & 2,78 & 0,02 & 0,10 & 2,90 & 102 & 0,20 & 1,29 & 6,20 & 9,29 & 0,78 \\
\hline 10 & 7,4 & 448,0 & 0,448 & 1,00 & 1,08 & 1,51 & 0,81 & 4,40 & 0,00 & 0,92 & 3,37 & 0,12 & 4,40 & 290 & 0,04 & 1,48 & 0,13 & 1,29 & 1,10 \\
\hline 11 & 7,2 & 378,5 & 0,379 & 0,83 & 0,86 & 1,32 & 0,69 & 3,70 & 0,00 & 0,43 & 3,23 & 0,03 & 3,70 & 234 & 0,05 & 1,44 & 2,83 & 5,50 & 0,94 \\
\hline 12 & 7,5 & 346,0 & 0,346 & 0,76 & 0,78 & 1,26 & 0,60 & 3,40 & 0,00 & 1,60 & 1,00 & 0,80 & 3,40 & 254 & 0,00 & 1,44 & 6,20 & 10,34 & 0,89 \\
\hline 13 & 7,4 & 365,5 & 0,366 & 0,82 & 0,84 & 1,28 & 0,66 & 3,60 & 0,00 & 1,67 & 1,07 & 0,87 & 3,60 & 236 & 0,05 & 1,40 & 6,30 & 10,26 & 0,92 \\
\hline 14 & 7,1 & 379,0 & 0,379 & 0,82 & 0,86 & 1,33 & 0,69 & 3,70 & 0,00 & 0,09 & 3,57 & 0,04 & 3,70 & 260 & 0,02 & 1,45 & 2,14 & 4,56 & 0,78 \\
\hline 15 & 5,1 & 385,5 & 0,386 & 0,71 & 0,75 & 1,31 & 1,03 & 3,80 & 0,00 & 0,59 & 3,17 & 0,04 & 3,80 & 146 & 0,02 & 1,53 & 6,20 & 11,04 & 0,96 \\
\hline 18 & 7,2 & 458,5 & 0,459 & 1,01 & 1,09 & 1,54 & 0,86 & 4,50 & 0,00 & 2,95 & 1,50 & 0,05 & 4,50 & 20056 & 0,00 & 1,50 & 6,20 & 10,82 & 1,08 \\
\hline 19 & 7,2 & 464,0 & 0,464 & 1,03 & 1,14 & 1,56 & 0,87 & 4,60 & 0,00 & 2,58 & 1,03 & 0,98 & 4,60 & 184 & 0,04 & 1,50 & 6,20 & 10,78 & 1,24 \\
\hline 20 & 7,2 & 433,0 & 0,433 & 0,98 & 1,04 & 1,47 & 0,81 & 4,30 & 0,00 & 3,23 & 0,65 & 0,42 & 4,31 & 176 & 0,06 & 1,46 & 6,20 & 10,53 & 1,08 \\
\hline 21 & 7,7 & 448,5 & 0,449 & 1,03 & 1,11 & 1,45 & 0,81 & 4,40 & 0,00 & 2,03 & 1,25 & 1,13 & 4,41 & 186 & 0,06 & 1,40 & 3,51 & 6,32 & 1,07 \\
\hline 22 & 7,4 & 457,0 & 0,457 & 1,06 & 1,13 & 1,47 & 0,85 & 4,51 & 0,00 & 4,37 & 0,12 & 0,02 & 4,50 & 178 & 0,07 & 1,40 & 6,20 & 10,11 & 1,00 \\
\hline 23 & 7,7 & 458,0 & 0,458 & 1,06 & 1,15 & 1,46 & 0,83 & 4,50 & 0,00 & 4,33 & 0,13 & 0,03 & 4,50 & 148 & 0,04 & 1,39 & 6,20 & 10,00 & 1,14 \\
\hline 24 & 7,7 & 290,0 & 0,290 & 0,68 & 0,66 & 1,06 & 0,50 & 2,90 & 0,00 & 2,83 & 0,05 & 0,02 & 2,91 & 204 & 0,09 & 1,29 & 3,67 & 6,04 & 0,75 \\
\hline 25 & 7,2 & 456,0 & 0,456 & 0,96 & 1,22 & 1,45 & 0,86 & 4,49 & 0,00 & 4,35 & 0,10 & 0,05 & 4,50 & 912 & 0,11 & 1,39 & 3,84 & 6,72 & 0,77 \\
\hline 26 & 7,1 & 2050,5 & 2,051 & 5,03 & 5,00 & 8,36 & 2,10 & 20,49 & 0,00 & 10,96 & 4,98 & 4,56 & 20,50 & 772 & 0,03 & 3,73 & 6,03 & 26,24 & 3,97 \\
\hline 27 & 6,9 & 1878,5 & 1,879 & 4,58 & 4,96 & 6,86 & 2,31 & 18,71 & 0,00 & 5,63 & 7,43 & 5,63 & 18,70 & 244 & 0,04 & 3,14 & 6,07 & 22,21 & 2,28 \\
\hline 28 & 7,4 & 527,5 & 0,528 & 1,14 & 1,43 & 1,57 & 1,06 & 5,20 & 0,00 & 4,37 & 0,47 & 0,37 & 5,20 & 184 & 0,03 & 1,38 & 6,20 & 9,97 & 1,17 \\
\hline 29 & 7,5 & 456,0 & 0,456 & 1,10 & 1,17 & 1,43 & 0,80 & 4,50 & 0,00 & 1,27 & 1,97 & 1,27 & 4,50 & 96 & 0,04 & 1,34 & 0,05 & 1,41 & 1,87 \\
\hline 30 & 7,2 & 518,0 & 0,518 & 0,91 & 0,99 & 2,33 & 0,86 & 5,09 & 0,00 & 4,87 & 0,17 & 0,07 & 5,10 & 168 & 0,13 & 2,39 & 6,20 & 17,21 & 3,31 \\
\hline 31 & 7,7 & 480,0 & 0,480 & 0,80 & 0,80 & 2,36 & 0,84 & 4,80 & 0,00 & 4,63 & 0,13 & 0,03 & 4,80 & 193 & 0,03 & 2,64 & 2,82 & 10,08 & 3,73 \\
\hline 32 & 7,3 & 253,5 & 0,254 & 0,34 & 0,38 & 1,42 & 0,36 & 2,50 & 0,00 & 1,88 & 0,13 & 0,48 & 2,50 & 256 & 0,07 & 2,37 & 6,20 & 17,04 & 1,01 \\
\hline $\begin{array}{l}\text { Dest. } \\
\text { Est. }\end{array}$ & 0,5 & 392,4 & 0,4 & 0,99 & 1,03 & 1,55 & 0,42 & 3,92 & 0,00 & 2,16 & 1,68 & 1,31 & 3,92 & 3501,26 & 0,05 & 0,60 & 2,05 & 6,01 & 0,84 \\
\hline Mínimo & 5,1 & 253,5 & 0,3 & 0,34 & 0,38 & 1,06 & 0,36 & 2,50 & 0,00 & 0,09 & 0,02 & 0,01 & 2,50 & 96,00 & 0,00 & 1,29 & $\overline{-} 13$ & 1,29 & 0,75 \\
\hline Máximo & 7,7 & 2050,5 & 2,1 & 5,03 & 5,00 & 8,36 & 2,31 & 20,49 & 0,00 & 10,96 & 7,43 & 5,63 & 20,50 & 20056,00 & 0,20 & 3,73 & 8,40 & 26,24 & 3,97 \\
\hline Moda & 7,5 & 290,0 & 0,3 & 0,82 & 0,66 & 1,06 & 0,81 & 4,50 & 0,00 & 2,83 & & & 4,50 & 184,00 & 0,00 & & 6,20 & & \\
\hline
\end{tabular}


Tabla 2. Determinaciones físico químicas del agua

\begin{tabular}{|c|c|c|}
\hline PARÁMETRO & MÉTODO & RFERENCIA \\
\hline $\mathrm{pH}$ & Potenciómetro ThermoCientific & $\begin{array}{l}\text { NMX-AA- } \\
\text { 008-SCFI- } \\
2000\end{array}$ \\
\hline $\begin{array}{c}\text { Conductividad } \\
\text { eléctrica }\end{array}$ & Potenciómetro ThermoCientific & $\begin{array}{c}\text { NOM-AA-93- } \\
1984\end{array}$ \\
\hline $\begin{array}{c}\text { Residuo seco } \\
\text { evaporado }\end{array}$ & Gravimetría estufa & $\begin{array}{c}\text { NOM-AA-34- } \\
1981\end{array}$ \\
\hline $\begin{array}{l}\text { Residuo seco } \\
\text { calcinado }\end{array}$ & Gravimetría mufla & $\begin{array}{c}\text { NOM-AA-34- } \\
1981 \\
\text { APHA, } 1995\end{array}$ \\
\hline Sodio y potasio & Espectrofotometría de absorción & $\begin{array}{c}3500-\mathrm{Na}^{+} \mathrm{y} \mathrm{K}^{+} \\
\mathrm{D}\end{array}$ \\
\hline $\begin{array}{l}\text { Calcio y } \\
\text { magnesio }\end{array}$ & Espectrofotometría de absorción & $\begin{array}{l}\text { APHA, } 1995 \\
3500 \mathrm{Ca}^{2+}, \mathrm{D}\end{array}$ \\
\hline Carbonatos & $\begin{array}{c}\text { Volumétrico titulación con ácido } \\
\text { sulfúrico (Merck Titrisol Art. } \\
\text { 9984) 0,01N indicador } \\
\text { fenoftaleína }\end{array}$ & $\begin{array}{c}\text { APHA, } 1995 \\
2320 \mathrm{~B}\end{array}$ \\
\hline Bicarbonatos & $\begin{array}{l}\text { Volumétrico titulación con ácido } \\
\text { sulfúrico (Merck Titrisol Art. } \\
\text { 9984) 0,01N. }\end{array}$ & $\begin{array}{c}\text { APHA, } 1995 \\
2320 \mathrm{~B}\end{array}$ \\
\hline Cloruros & $\begin{array}{l}\text { Mohr. Titulación con nitrato de } \\
\text { plata (Merck solución Art. } \\
\text { OC248386) } 0,01 \mathrm{~N}\end{array}$ & $\begin{array}{l}\text { APHA, } 1995 \\
4500-C-I B\end{array}$ \\
\hline Sulfatos & EspectrofotometríaHach $\lambda=450 \mathrm{~nm}$ & $\begin{array}{c}\text { Método } 375.4 \\
\text { EPA }\end{array}$ \\
\hline
\end{tabular}

\section{RESULTADOS Y DISCUSIÓN}

\subsection{Distribución de la salinidad de las aguas de riego}

La conductividad eléctrica $\mathrm{CE}$ al igual que los residuos sólidos totales, son estimadores cuantitativos de la concentración de sales disueltas en las aguas de riego (Richard et al., 1959), para el $25 \%$ de las muestras la CE se encuentra entre 300 y $400 \mu \mathrm{S}$ $\mathrm{cm}^{-1}$, el $31,35 \%$ entre 400 y $500 \mu \mathrm{S} \mathrm{cm}^{-1}$ y un $12,50 \%$ entre 600 y $700 \mu \mathrm{S} \mathrm{cm} \mathrm{cm}^{-1}$. Para el laboratorio de Salinidad de Riverside (USA) la mayoría de aguas para riego del cantón están clasificadas como $\mathrm{C} 2$, aguas de salinidad media que pueden usarse para riego bajo la condición de que exista por lo menos un lavado moderado de los suelos. Un porcentaje importante está clasificada como C4 de salinidad muy alta que en general no son recomendadas para riego, se pueden usar en suelos muy permeables y con buen drenaje que podría ser el caso del cantón. La Figura 3 muestra la distribución de la salinidad en base a la conductividad eléctrica del cantón.

\section{2 Índices de salinidad IS}

Las sales tienen un efecto adverso sobre las propiedades físicas, químicas y biológicas del suelo. La salinidad en los suelos es un problema para la agricultura del mundo, siendo el factor que más limita la productividad de los cultivos (Gili et al., 2003).

Las aguas subterráneas constituyen la fuente de riego más utilizada en el cantón Milagro que se caracteriza por su alto contenido de bicarbonatos, las aguas de este tipo tienden a generar precipitados de calcio y magnesio y como consecuencia, incrementan la RAS del suelo.

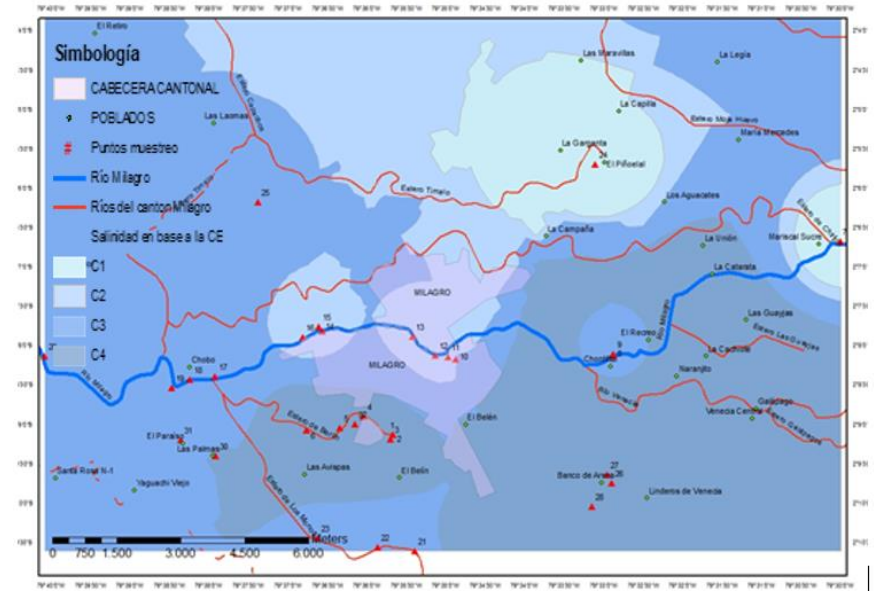

Figura 3. Distribución espacial de la salinidad en base a la conductividad eléctrica ( $\mu \mathrm{S} \mathrm{cm-1)} \mathrm{(Richard} \mathrm{et} \mathrm{al.,} \mathrm{1959)} \mathrm{de} \mathrm{la} \mathrm{microcuenca} \mathrm{del} \mathrm{río} \mathrm{Milagro.}$

La salinidad efectiva es una estimación más real del peligro que presentan las sales solubles del agua de riego al formar parte de la solución del suelo, pues considera la precipitación de los carbonatos de calcio y magnesio. Este proceso es más relevante cuando las aguas tiene un alto contenido de carbonatos y bicarbonatos (Uvalle et al., 2000). Los resultados del índice de salinidad efectiva se muestran en el Tabla 3. Un porcentaje elevado $43,75 \%$ de las aguas de riego está condicionada para su uso, pudiendo causar graves problemas a los cultivos y a los suelos.

En la Tabla 4 se puede observar los resultados de la salinidad potencial, que es un índice para determinar el peligro de cloruros y parte de sulfatos a bajos niveles de humedad, por debajo del $50 \%$ y que por lo tanto aumentan considerablemente la presión osmótica (Uvalle et al., 2000). Se demuestra que el porcentaje de restricciones es de $28,13 \%$, aunque no hay aguas que no sean recomendables no se puede descartar la concentración de iones y la posible sodificación del suelo.

\section{3 Índices de salinidad IS}

La sodicidad mide el efecto probable de sodio sobre las propiedades físicas del suelo. Cuando el sodio se encuentra en altas concentraciones en el agua de riego y el calcio y el magnesio precipitan en la solución del suelo por la acción de carbonatos y bicarbonatos, entonces el sodio se acumula y substituye al calcio y al magnesio en el intercambio de cationes, dando lugar a un desequilibrio eléctrico de las partículas coloidales del suelo por el predominio de cargas negativas, las partículas se repelen, el suelo se deflocula y pierde estructura, se fomenta la compactación y encostramiento, afectando el desarrollo normal de los cultivos (Uvalle et al., 2000).

La RAS de las aguas están íntimamente relacionada con la relación de adsorción de sodio de la solución del suelo, es considerado un buen indicador del riesgo de sodificación del suelo y por lo tanto puede deducirse el porcentaje de sodio intercambiable PSI esperable en el largo plazo (Prieto, 2008). 
Tabla 3. Clasificación de las aguas del cantón Milagro en base a la salinidad

\begin{tabular}{cccccc}
\hline \multicolumn{5}{c}{ efectiva } \\
No. & $\begin{array}{c}\text { Índice de } \\
\text { Salinidad } \\
\text { Efectiva }\end{array}$ & $\begin{array}{c}\text { Clasificación } \\
\text { Salinidad } \\
\text { Efectiva }\end{array}$ & No. & $\begin{array}{c}\text { Indice de } \\
\text { Salinidad } \\
\text { Efectiva }\end{array}$ & $\begin{array}{c}\text { Clasificación } \\
\text { Salinidad } \\
\text { Efectiva }\end{array}$ \\
\hline 1 & 3,782 & Condicionada & 17 & 2,119 & Buena \\
2 & 2,608 & Buena & 18 & 2,399 & Buena \\
3 & 3,611 & Condicionada & 19 & 2,434 & Buena \\
4 & 4,475 & Condicionada & 20 & 2,280 & Buena \\
5 & 4,233 & Condicionada & 21 & 2,375 & Buena \\
6 & 5,300 & Condicionada & 22 & 2,317 & Buena \\
7 & 2,617 & Buena & 23 & 2,292 & Buena \\
8 & 2,895 & Buena & 24 & 1,562 & Buena \\
9 & 1,554 & Buena & 25 & 2,315 & Buena \\
10 & 3,399 & Condicionada & 26 & 10,466 & Condicionada \\
11 & 3,233 & Condicionada & 27 & 13,067 & Condicionada \\
12 & 1,866 & Buena & 28 & 2,636 & Buena \\
13 & 1,941 & Buena & 29 & 3,233 & Condicionada \\
14 & 3,567 & Condicionada & 30 & 3,192 & Condicionada \\
15 & 3,167 & Condicionada & 31 & 3,204 & Condicionada \\
16 & 2,017 & Buena & 32 & 1,783 & Buena \\
\hline
\end{tabular}

Tabla 4. Clasificación de las aguas del muestreo de las aguas del cantón Milagro en base a la salinidad potencial.

\begin{tabular}{cccccc}
\hline No. & $\begin{array}{c}\text { Índice de } \\
\text { Salinidad } \\
\text { Potencial }\end{array}$ & $\begin{array}{c}\text { Clasificación } \\
\text { Salinidad } \\
\text { Potencial }\end{array}$ & No. & $\begin{array}{c}\text { Índice de } \\
\text { Salinidad } \\
\text { Potencial }\end{array}$ & $\begin{array}{c}\text { Clasificación } \\
\text { Salinidad } \\
\text { Potencial }\end{array}$ \\
\hline 1 & 1,150 & Buena & 17 & 0,125 & Buena \\
2 & 2,558 & Buena & 18 & 1,525 & Buena \\
3 & 1,288 & Buena & 19 & 1,525 & Buena \\
4 & 3,513 & Condicionada & 20 & 0,863 & Buena \\
5 & 3,400 & Condicionada & 21 & 1,813 & Buena \\
6 & 4,050 & Condicionada & 22 & 0,125 & Buena \\
7 & 2,688 & Buena & 23 & 0,150 & Buena \\
8 & 2,100 & Buena & 24 & 0,063 & Buena \\
9 & 0,067 & Buena & 25 & 0,125 & Buena \\
10 & 3,425 & Condicionada & 26 & 7,263 & Condicionada \\
11 & 3,250 & Condicionada & 27 & 10,250 & Condicionada \\
12 & 1,400 & Buena & 28 & 0,650 & Buena \\
13 & 1,500 & Buena & 29 & 2,600 & Buena \\
14 & 3,588 & Condicionada & 30 & 0,200 & Buena \\
15 & 3,188 & Condicionada & 31 & 0,150 & Buena \\
16 & 0,837 & Buena & 32 & 0,375 & Buena \\
\hline
\end{tabular}

Las formulaciones de RAS más frecuentemente utilizados en el manejo de aguas y suelos son RAS $_{\text {org, }}$ RAS $_{\text {aj }}$ y RAS ${ }^{\circ}$. El índice de RAS original no toma en cuenta los procesos de dilución y precipitación que se producen en el suelo por dilución de la solución del suelo o presencia de $\mathrm{CO}_{2}$, o la presencia de abundantes cantidades de calcio, carbonatos $\mathrm{CO}_{3}$ 2, bicarbonatos $\mathrm{HCO}_{3}^{-}$o sulfatos $\mathrm{SO}_{4}^{-2}$. Para esto el Laboratorio de Salinidad del USDA (1951), propuso el Carbonato de Sodio Residual (CSR), con el mismo objetivo Ayers y Westcot (1976) introdujeron el concepto de RAS ajustado $\left(\mathrm{RAS}_{\mathrm{aj}}\right.$.) propuesto originalmente por Rhoades (1972), en cuyo cálculo se tiene en cuenta la concentración de $\mathrm{CO}_{3}^{-2}$ y $\mathrm{HCO}_{3}^{-}$y no toma en cuenta la precipitación del calcio y del magnesio.

La Figura 4 muestra el mapa de distribución espacial de las aguas con respecto a la RAS ajustada y la Figura 5 muestra la clasificación según Richard et al., (1959), el 42,87\% de las aguas pertenecen a la clasificación S1 (poco sodio), 46,88\% son S2 que indica un alto porcentaje de aguas con contenido de sodio medio, lo que representa un peligro en suelos de textura fina con alta capacidad de intercambio catiónico. También hay aguas clasificadas como S3, altas en sodio con peligro de acumulación en el suelo e incluso aguas muy altas en sodio $\mathrm{S} 4$.

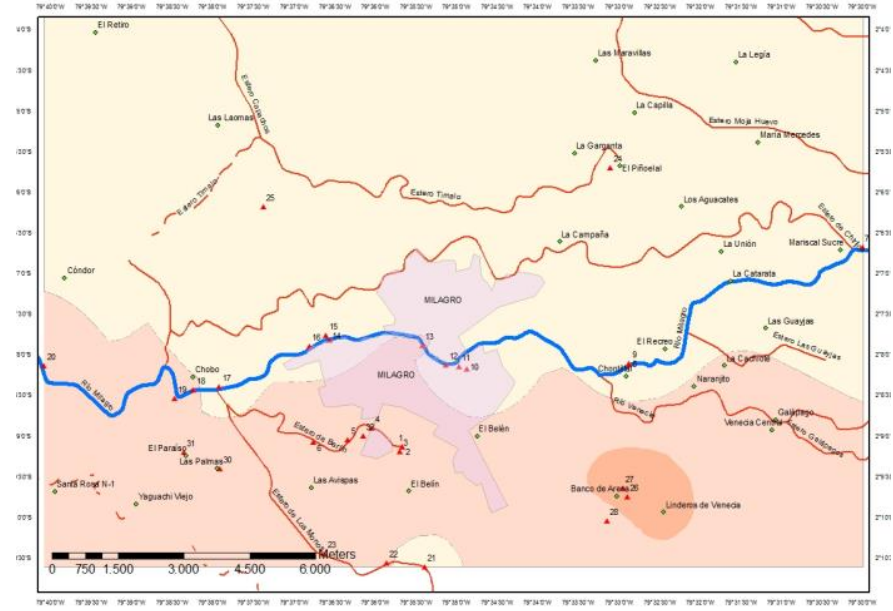

Figura 4. Distribución espacial de la salinidad del agua de la microcuenca del río Milagro en base al $\mathrm{RAS}_{\mathrm{aj}}$,

La RAS del agua de riego nos permite inferir la relación del porcentaje de sodio intercambiable PSI de los suelos para las condiciones de saturación en equilibrio con las aguas de riego.

Según Carrera (2009), el PSI se deriva de la ecuación de intercambio de Gapón, para la que los valores de coeficiente de selectividad iónica o constante de Gapón $\left(\mathrm{K}_{\mathrm{G}}\right)$, que representa la pendiente de la ecuación de regresión RAS-PSI, tienen valores de $\mathrm{K}_{1}=0,0072444, \mathrm{~K}_{2}=0,011861$ y $\mathrm{K}_{3}=$ $0,0168999\left(\mathrm{mmol} \mathrm{L}^{-1}\right)^{-1 / 2} \cdot \mathrm{K}_{\mathrm{G}}$ expresa directamente la complejidad de las estructuras cristalo-químicas de los sistemas arcillosos y orgánicos coloides-dispersos de los suelos (Uvalle et al., 2000).

La Figura 6 muestra los resultados de la relación para las todas las modificaciones de RAS.

El PSI es el parámetro que mejor puede correlacionarse con la posible alteración del estado estructural del suelo (disminuyendo su permeabilidad) y con los efectos tóxicos en los cultivos debido al sodio. Por ello una evaluación racional del riesgo de sodicidad potencial de un agua debe realizarse en base a un parámetro que se correlacione de forma satisfactoria con el PSI del suelo que resulta del riego con esa agua. La RAS corregida es la que, aparentemente, mejor se correlaciona con el PSI del suelo, por lo que aparece como el parámetro más real de determinación del riesgo de sodicidad de un agua de riego (Suarez, 1981). La Figura 7 muestra el diagrama de clasificación de las aguas de riego de la microcuenca del río Milagro en base a la RAS $^{\circ}$ (Richard, 1962). 


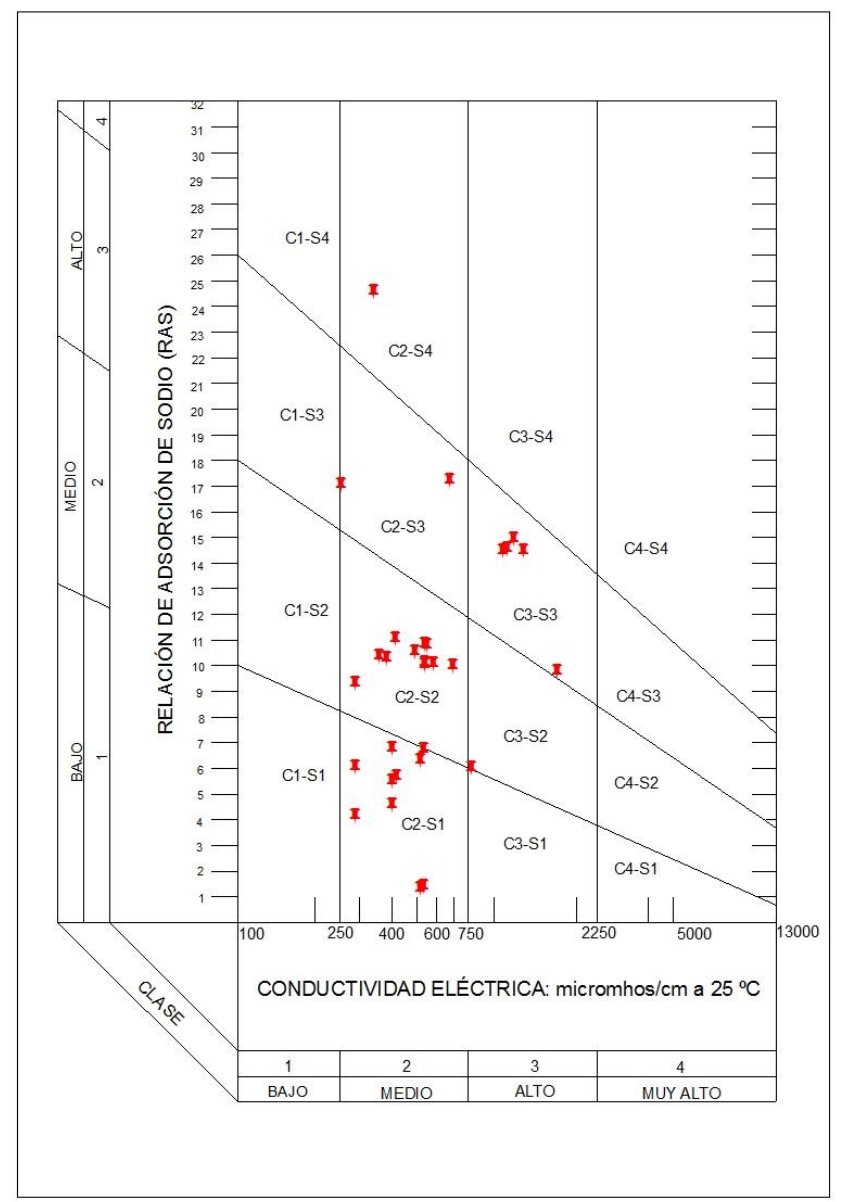

Figura 5. Diagrama de clasificación de las aguas para riego citada por Richards et al. (1959), $\left(\right.$ RAS $_{\text {aj }}$ ).

3.3 Efecto de las concentraciones de Carbonatos y Bicarbonatos

El exceso de carbonatos y bicarbonatos en las aguas de riego, pueden aumentar el índice de RAS ya que al combinarse con el calcio y magnesio presentes precipitan rápidamente, pues 1E10-9 y $1 \mathrm{E} 10-8$ a $25^{\circ} \mathrm{C}$ respectivamente (Chang, 2010), por cada 100 gramos de agua se mantienen disueltos apenas 0,0013 gramos de carbonato, por lo tanto al superar este valor rápidamente precipitan las sales, aumentando la proporción relativa de sodio presente en el suelo y el riego de sodificación del mismo (Martínez, 2009).

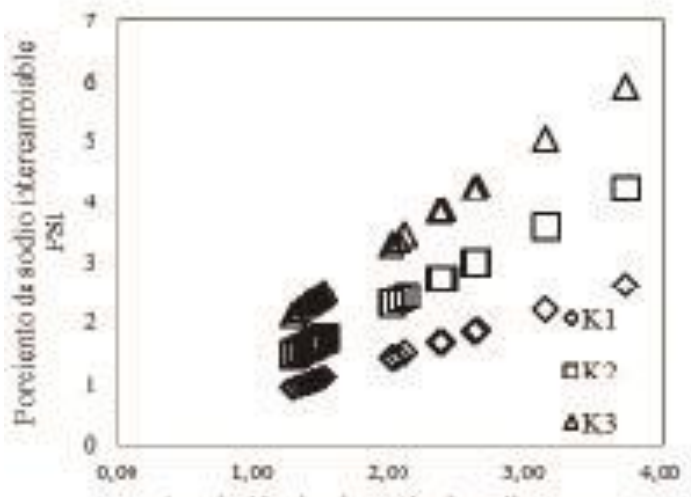

a) Rebción de adsrocín de sodio RA5 erie
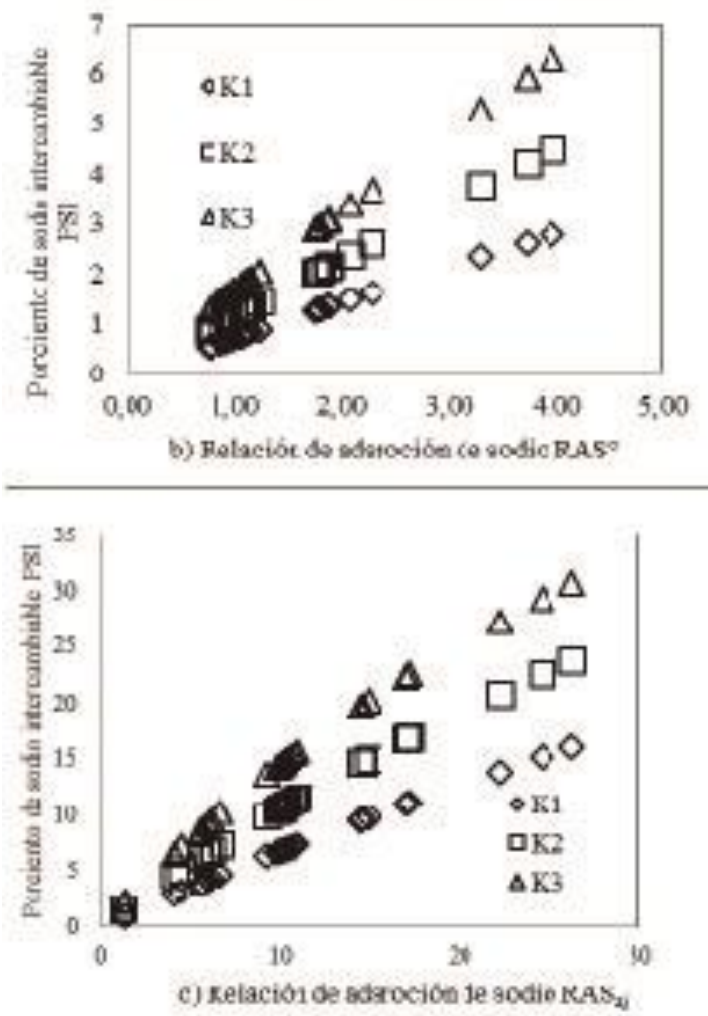

Figura 6. Relación PSI - RAS; a) RAS ${ }_{\text {orig }}$; b) $\operatorname{RAS}^{\circ}$; c) RAS $_{\text {aj }}$ 


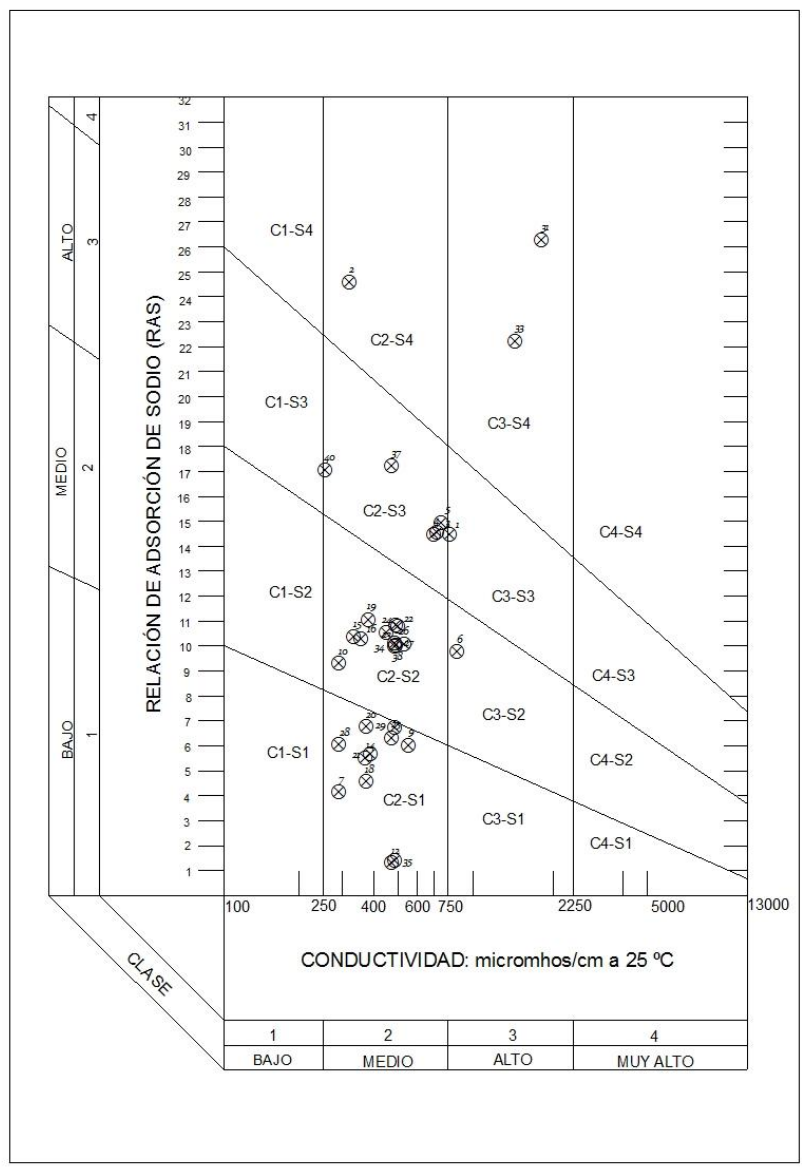

Figura 7. Diagrama de clasificación de las aguas para riego citada por Richards et al. (1959), de la microcuenca del río Milagro $\left(\mathrm{RAS}^{\circ}\right)$.

\section{CONCLUSIONES}

A través de las mediciones de algunos parámetros en el agua de riego, se podría predecir el estado del suelo de cultivo. En el agua para riego se pueden medir con relativa facilidad parámetros como los sólidos totales disueltos, conductividad eléctrica, $\mathrm{pH}$ entre otros, que indirectamente pueden proporcionar las propiedades físico-químicos del suelo, que de otra manera al hacer las pruebas en el suelo tomaría más tiempo y los costos serían más altos.

La RAS de las aguas del cantón Milagro es alta en época de estiaje, por lo tanto no se debe permitir que el suelo se seque, para evitar que precipiten carbonatos de calcio y magnesio, así pues la frecuencia de riego debe aumentar para contrarrestar que la solución del suelo pierda los cationes alcalinotérreos y la consecuente reducción de la productividad agrícola.

Es necesario que exista un lavado profundo de los suelos especialmente después de la época de baja precipitaciones para evitar la sodificación de los suelos y sus efectos sobre los cultivos, de tal manera de sustituir el sodio intercambiable por iones calcio o hacer tratamientos más intensos con yeso, esto mantendrá al calcio y magnesio en solución en relación al sodio. Este trabajo dará buenos resultados pues el drenaje del cantón Milagro es suficiente por su contenido de material deposicional con un buen contenido de limos y arenas no consolidadas.
La calidad del agua y su influencia sobre los suelos de cantón Milagro fluctúa periódicamente, esto se confirma de acuerdo a las mediciones de CE en las aguas que en promedio es de 560 $\mu \mathrm{S} \mathrm{cm}^{-1}$ que supera significativamente a lo recomendable por las normas ambientales, en comparación con la conductividad eléctrica en época de lluvias que alcanza un nivel inferior a 100 $\mu \mathrm{S} \mathrm{cm}^{-1}$ (Carrera, 2013). El pH promedio es de 7,3 lo que indica que hay condiciones de neutralidad, sin embargo existe un punto en el que el $\mathrm{pH}$ es de 5,2 punto en el que se mezcla el agua de riego con vinaza, lo que sin el control adecuado, puede causar problemas de acidificación del suelo y la eutrofización de las aguas.

Es recomendable que los suelos del cantón se sometan a un examen por lo menos una vez al año para determinar los posibles problemas relacionados con el sodio y proceder a tomar medidas de remediación en lugar de usar excesivas cantidades de fertilizantes en aras de mejorar el rendimiento de los cultivos.

\section{AGRADECIMIENTO}

A la Universidad de las Fuerzas - ESPE que permitió el trabajo en los laboratorios de la Carrera de Biotecnología y el CENCINAT.

\section{REFERENCIAS}

APHA, -A.-W. (1995). American Public Health Association,. Standard methods for the examination of water and wastewater 19th edition. Publication office American Public Health Association, Chapter 1 p. 45; Chapter 2 p. 88; Chapter 3 p. 10. Washington D. C., USA.

Ayers, R y Westcot, D. (1987). La calidad del agua y su uso en la agricultura. Estudio FAO Riego y Drenaje 29 Rev. 1. Traducido al español por J. F. Alfaro de: Water quality and use in agriculture. FAO, Roma, $175 \mathrm{pp}$

Carrera, D. (2009). Salinidad en suelos y aguas superficiales y subterráneas de la cuenca evaporítica del río Verde-Matehuala, San Luis Potosí, Tesis Doctoral, Tesococo, pp. 334. . Montecillo, Dto. México, México.

Carrera, D., Pérez, A. (2013). Salinidad, Fosfatos, Nitratos y problemas de infiltración en las aguas de Milagro, Ecuador, 9. Ciencia, 102.

Chang, R. (2010), Química, México: Mc Graw Hill.

CLIRSEN. (2009). Centro de Levantamientos Integrados de Recursos Naturales por Sensores Remotos. Generación de geoinformación para la gestión del territorio y valoración de tierras rurales de la cuenca del río guayas escala 1:25 000. SENPLADES. Quito, Ecuador.

INAMHI (2013). Anuarios Meteorológicos publicados por el Instituto Nacional de Meteorología e Hidrología. Quito, Ecuador.

IGM. (2009). Mapa Físico del Ecuador, escala 1:1 000 000. Quito, Ecuador: IGM.

Lenntech (n.d.). Calidad del agua para irrigación. Obtenido de: http://www.lenntech.es (marzo, 2017).

Martínez, L. (n.d.). Estudio de la calidad agronómica del agua de riego de las Islas Baleare, España.Obtenido de: http://dgrechid.caib.es/www/doc/AIGUES_SUBTERRANIES_1.pdf. (Noviembre, 2014).

Método 375.4 EPA (1978). Método turbidimétrico para determinación de sulfatos.

NMX-AA-008-SCFI-2000. Norma Mexicana. Análisis de Agua Determinación del $\mathrm{pH}$ - Método de Prueba.

NOM-AA-93-1984. Norma Mexicana. Análisis de Agua - Determinación de la Conductividad Electrolítica - Método de Prueba.

NOM-AA-34-1981. Norma Mexicana. Análisis de Agua - Determinación de sólidos y sales disueltas en aguas naturales, residuales y residuales tratadas - método de prueba.

Prieto, D. (2008). Riego con aguas salinas y aguas de drenaje, control de impacto de salinidad, Red Riegos. Jornadas sobre "Ambiente y Riegos: Modernización y Ambientalidad”. La Antigua (Guatemala). CYTED y AECID. 
PDOTM (2014). Plan de Desarrollo y Ordenamiento Territorial del Cantón Milagro Obtenido de: http://www.guayas.gob.ec/cantones/milagro. (Marzo 2017)

Richards, L., Allison, L., Bernstein, C., Bower, J., Brown, M., Fireman, J., Harcher, H., Hayward, G., Pearson, R., \& Reeve 1, A. (1959). Suelos Salinos y Sódicos. Personal del Laboratorio de Salinidad de los Estados Unidos de América. No. 60. Manual de Agricultura, 172.

Richards, L. (1962) Diagnóstico y rehabilitación de suelos salinos y sódicos. Editor México: Editorial Cultura.

Uvalle. (2000). Memorias del curso de análisis de agua, suelo y planta, su interpretación y utilidad agrícola, Capítulo XI., p 8. Calidad del agua para riego agrícola (pág. 8). Guayaquil: FUNPROVER.

Torres, A., \& Acevedo, E. (2008). El problema de la salinidad en los recursos suelo y agua que afectan el riego y cultivos en los valles de Lluta y Azapa en el norte de Chile. IDESIA, 26(3), 31-44. https://scielo.conicyt.cl/scielo.php?script=sci_arttext\&pid=S0718$\underline{34292008000300004}$

Chen, W. (2010) Evaluating salinity distribution in soil irrigated with saline water in arid regions Agric. Water Manage. 97 2001-8

Gili, S., Marando, I., Irisarri, E., \& Sagardoy, R. (2003). Efecto de las técnicas de lavado y fertilización sobre la salinidad en suelos del alto valle de río Negro y Neuquén, Argentina, Agricultura Técnica, 64(3), 295-304. https://scholar.google.com.ec/scholar?q=Agricultura+T\%C3\%A9cnica,+ 64(3),+295-304.\&hl=es\&as $\mathrm{sdt}=0$ \&as vis=1\&oi=scholart

USDA. (2013). United States Department of Agriculture.

Suarez, D., (1981). Relation between pHcand Sodium Absortion Ratio (SAR) and an alternate method of estimating SAR of soil or drainage Waters, , 45,. Soil Science Society of America, 469-475

\section{BIOGRAFÍA}

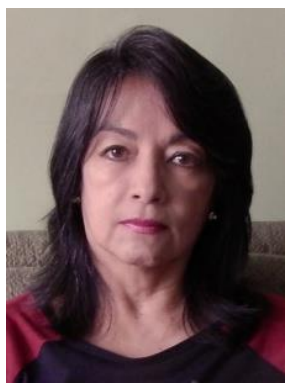

Sonia Alexandra Pérez Molina. Nacida en Quito el 18 de diciembre de 1962, Estudios primarios en la Escuela Franciscana "Alvernia" y los secundarios en el Colegio "Santa María Eufrasia".

Ingeniera Química de la Escuela Politécnica Nacional y Máster en Sistemas de Gestión Ambiental por la Universidad de las Fuerzas Armadas ESPE. Vida profesional en docencia, con grandes satisfacciones en el proceso en varias instituciones de prestigio, como: Colegio Técnico de Aviación Civil, Escuela Militar "Eloy Alfaro", Escuela Politécnica Nacional y la Universidad de las Fuerzas Armadas - ESPE. 